\title{
A MIXING DYNAMICAL SYSTEM ON THE CANTOR SET
}

\author{
JEONG H. KIM \\ Applied Mathematical Sciences \\ Ames Laboratory \\ Iowa State University \\ Ames, IA 50011
}

(Recened March 17, 1994 and in revised form June 27,1994)

\begin{abstract}
In this paper we give mixıng properties (ergodic, weak-mixing and strong-mixing) to a dynamical system on the Cantor set by showing that the one-sided $\left(\frac{1}{2}, \frac{1}{2}\right)$-shift map is isomorphic 10 a measure preserving transformation defined on the Cantor set.
\end{abstract}

KEY WORDS AND PHRASES: Dynamical system, Mixing, Cantor set. 1991 AMS SUBJECT CLASSIFICATION CODES: 28D 10

\section{INTRODUCTION}

A dynamical system is a quadruple $(X, \mathcal{A}, m, T)$, where $X$ is a non-empty space, $\mathcal{A}$ is a $\sigma$-algebra of subsets of $X, m$ is a measure defined on $\mathcal{A}$ and $T$ is a measure-preserving transformation on $X$. Ergodic theory may be defined to be the study of transformations or groups of transformations, which are defined on some measure space, which are measurable with respect to the measure structure of that space, and which leave the measure of all measurable subsets of the space. If arbitrary orbit of transformation of a given dynamical system passes through every point of this system, it is said that this dynamical system satisfies the ergodic hypothesis. The ergodic hypothesis was introduced by L. Boltzman and W. Gibbs to establish the following principle:

$$
\lim _{n \rightarrow \infty} \frac{1}{n} \sum_{h=0}^{n-1} f\left(T^{h} x\right)=\frac{1}{m(X)} \int_{X} f(x) d m
$$

which says that the time mean of $f$ is equal to the space (or phase) mean of $f$. Many contrarguments about this hypothesis came out but in $1912 \mathrm{H}$. Poincare proved the so-called recurrence theorem and in 1931 G. D. Birkhoff and J. Von Neumann proved the existence of time mean and thus this hypothesis was accepted. This is the historical beginning of mathematical study of ergodic theory. The study of ergodic theory can be categorized into one of four types, that is, (i) Measure theoretic, (ii) Topological, (iii) Mixture of (i) and (ii), and (iv) Smooth. In this paper we are concerned about measure theoretic type and we shall assume that the measure is finite and normalized to have total measure one (the probability measure). We refer [1], [2] as general texts for ergodic theory.

The main purpose in this paper is to give ergodic property to a dynamical system on the Cantor ternary set. In Section 2 we first summarize properties of the generalized Cantor set and the Cantor measure. In Section 3 we introduce mixing properties (i.e. strong-mixing, weak-mixing and ergodic properties) for measure preserving transformations. The one-sided shift map is shown to satisfy these properties. In Section 4 we show that the one-sided shift transformation is isomorphic to a measure preserving transformation defined on the Cantor ternary set so that a dynamical system on the Cantor set has the three mixing properties.

\section{THE GENERALIZED CANTOR SET AND THE CANTOR MEASLRE}

In this section we summarize some properties of the generalized Cantor set and the Cantor measure. We first define the Cantor set, the Cantor function and the Cantor measure. We describe the Lebesque measure of the Cantor set together with the topological properties of the set and the relation 
hetween the Cantor measure and the Lebesque measure of the set. The Cantor set is known to have the same cardinality as the interval $[0,1]$. Finally in this rection we present the ternary representation of Cantor's middle third set.

DEFINITION 2.1. Let $n_{1}, n_{2}, \ldots, n_{k}, \ldots$ be a requence of real numbers such that $1<n_{k}<\infty$ for every $k=1,2, \ldots$. We define the generalized Cantor set denoted by $C\left(n_{1}, n_{2}, \ldots n_{k} \ldots\right)$ (or briefly $C)$ as follows: We remove the open interval of size $\frac{1}{n}$ about the middle pount $\frac{1}{2}$ from the interval $[0,1]$. Then remove the open interval of size $\frac{1}{2}\left(1-\frac{1}{n_{1}}\right) \frac{1}{n_{2}}$ about the middle of each of the two remaining closed intervals. After $k$ removals, there are $2^{k}$ closed intervals left denoted by $C^{\prime}\left(n_{k}\right), j=1,2,3, \ldots, 2^{k}$. and at the $k+1$ step we remove the open intervals of size $\frac{1}{2^{k}} \Pi_{t=1}^{\lambda}\left(1-\frac{1}{n_{t}}\right) \frac{1}{n_{k+1}}$ about the center of these intervals. The generalized Cantor set $C$ is defined to be the complement in the interval $[0,1]$ of the union of these open intervals, i.e.,

$$
C=\bigcap_{h=1}^{\infty} \bigcup_{j=1}^{2^{k}} C^{\prime}\left(n_{k}\right)
$$

which depends on the choice of the sequence $n_{1}, n_{2}, \ldots, n_{k}, \ldots$

DEFINITION 2.2. We define the Cantor function $F(t), t \in[0,1]$, by setting it equal to $1 / 2$ on the first interval removed, $1 / 2^{3}$ and $3 / 2^{2}$ on the two intervals removed at the second step, $1 / 2^{3}, 3 / 2^{3}, 5 / 3^{3}$ and $7 / 2^{3}$ on the third removal, etc. The values are chosen in the obvious way so that the function $F$ is monotone non-decreasing on $[0,1]$ ( $F$ is extended to $[0,1]$ by the continuity).

DEFINITION 2.3. The Cantor measure $\mu_{F}$ on $[0,1]$ is defined by

$$
\mu_{F}([0, t))=F(t)
$$

where $F(t)$ is the Cantor function.

We note that the above $\mu_{F}$ becomes a Borel measure by the following argument: If we let $\mathcal{A}$ be the semialgebra consisting of all intervals of the form $[a, b), 0 \leq a<b \leq 1$, and set $\mu_{F}([a, b))=F(b)-F(a)$, then $\mu_{F}$ satisfies the following two conditions:

1. $\mu_{F}(B)=\sum_{1=1}^{n} \mu_{F}\left(B_{l}\right)$ for any finite disjoint union $B\left(=\cup_{1=1}^{n} B_{\imath}\right)$ of $B_{1}$ 's in $\tilde{\mathcal{A}}$.

2. $\mu_{F}(B) \leq \sum_{i=1}^{\infty} \mu_{F}\left(B_{i}\right)$ for any countable disjoint union $B\left(=\cup_{i=1}^{\infty} B_{i}\right)$ of $B_{i}$ 's in $\dot{\mathcal{A}}$

Then $\mu_{F}$ admits a unique extension to a measure on the algebra generated by $\overline{\mathcal{A}}$ and thus, from Caratheodory extension theorem, $\mu_{F}$ can be extended to a $\sigma$-algebra $\mathcal{A}$ containing $\dot{\mathcal{A}}$. Hence we have an extension of $\mu_{F}$ to a Borel measure.

In the following we describe the topological structure of the generalized Cantor set $C$ and the relation between the Cantor measure and the Lebesque measure of the Cantor set.

1. There is a bijection map between the Cantor set $C$ and the interval $[0,1]$.

2. The Cantor set $C$ is always nowhere dense.

3. The Cantor set $C$ is a perfect set.

4. The Lebesque measure of the Cantor set $C$ is given by the infinite product $\pi_{k=1}^{\infty}\left(1-\frac{1}{n_{k}}\right)$.

5. The Cantor measure $\mu_{F}$ of the Cantor set $C$ is absolutely continuous with respect to the Lebesque measure of the set if $\sum_{k=1}^{\infty} \frac{1}{n_{k}}<\infty$.

6. The Cantor measure $\mu_{F}$ is mutually singular with respect to the Lebesque measure if $\sum_{k=1}^{\infty} \frac{1}{n_{k}}=\infty$.

If $n_{k}$ is a fixed positive rational number (constant disection ratio) for all $k=1,2, \ldots$, then we can 
obtan the concrete representatuon of the Cantor set (c) $|3|$ ). If in particular $n_{k}=3$ for all $k=1,2, \ldots$ in the definition of the generalused Cantor set $C$, then $1 \in C$ if and only if $x$ has a ternary

epresentation of the form $2 \sum_{n}, \frac{a_{n}}{z^{n}}$, where $a_{n} \in\{0,1\}$ for all $n=1.2$. In thes case. the set $C$ is called the Cantor ternary set or Cantor's muddle thurd set and the Cantor function $F$ on $C$ is given by

$$
F\left(2 \sum_{n-1}^{\infty} \frac{a_{n}}{3^{n}}\right)=\sum_{n-1}^{\infty} \frac{a_{n}}{2^{n}}, \quad a_{n} \in\{0,1\} .
$$

\section{DYNAMICAL SYSTEM AND THE ONE-SIDED SHIFT TRANSFORMATION}

In this section we define a measure preserving transformation and a dynamical system in terms of thıs transformation. We introduce the three kinds of mixing properties ( $1 \mathrm{c}$. ergodic, weak-mixing and strong-mixing) of a measure preserving transformation. The one-sided shift map is shown to be a transformation which has all of these three properties.

DEFINITION 3.1. Suppose the triples $\left(X_{1}, \lambda_{1}, m_{1}\right)$ and $\left(X_{2}, \mathcal{A}_{2}, m_{2}\right)$ are probability spaces. Then

(a) $T: X_{1} \rightarrow X_{2}$ is measurable if $T^{-1} \mathfrak{A}_{2} \subset \mathfrak{A}_{1}$.

(b) $T: X_{1} \rightarrow X_{2}$ is measure-preserving if $T$ is measurable and $m_{1}\left(T^{-1}(B)\right)=m_{2}(B)$ for all $B \in \mathcal{A}_{2}$.

The following theorem is well known in measure theory [4]. It provides the sufficient condition for the map $T$ to be measure-preserving in terms of generating algebra.

THEOREM 3.1. Suppose $\left(X_{1}, \mathcal{A}_{1}, m_{1}\right)$ and $\left(X_{2}, \mathcal{A}_{2}, m_{2}\right)$ are probability spaces. Let $\mathcal{A}_{2}^{*}$ be an algebra which generates the $\sigma$-algebra $\mathcal{A}_{2}$. If $T$ is a map such that, for any $B \in \mathcal{A}_{2}^{*}, T^{-1}(B) \in \mathcal{A}_{1}$ and $m_{1}\left(T^{-1}(B)\right)=m_{2}(B)$, then $T$ is measure-preserving.

Since we wish to study the iteration $T^{n}$ of the transformation $T$, we shall deal with the identical case $\left(X_{1}, \mathcal{A}_{1}, m_{1}\right)=\left(X_{2}, \mathcal{A}_{2}, m_{2}\right)$. A probability space $(X, \mathcal{A}, m)$ together with a measure-preserving transformation $T$ defines a dynamical system $(X, \mathcal{A}, m, T)$. The above Theorem 3.1 shows that whether a given transformation is measure-preserving or not is determined only by the knowledge of an algebra generating the $\sigma$-algebra $\mathcal{A}$.

In the following we define the three mixing properties for a dynamical system. In the theory of dynamical systems, mixing is the property of indecomposibility of a dynamical system into nontrivial invariant subsets.

DEFINITION 3.2. $(X, \mathcal{A}, m, T)$ is a dynamical system.

(a) $T: X \rightarrow X$ is ergodic if for arbitrary $A, B \in \mathcal{A}$

$$
\frac{1}{n} \sum_{k=0}^{n-1} m\left(T^{-k} A \cap B\right) \rightarrow m(A) m(B) \quad \text { as } \quad n \rightarrow \infty .
$$

(b) $T: X \rightarrow X$ is weak-mixing if for arbitrary $A, B \in \mathcal{A}$

$$
\frac{1}{n} \sum_{k=0}^{n-1}\left|m\left(T^{-k} A \cap B\right)-m(A) m(B)\right| \rightarrow 0 \quad \text { as } n \rightarrow \infty .
$$

(c) $T: X \rightarrow X$ is strong-mixing if for arbitrary $A, B \in \mathcal{A}$

$$
m\left(T^{-n} A \cap B\right) \rightarrow m(A) m(B) \text { as } n \rightarrow \infty .
$$

REMARK. (i) It can be shown [1] that $T: X \rightarrow X$ is ergodic if and only if, for arbitrary $A \in \mathcal{A}, T^{-1}(A)=(A)$ implies that either $m(A)=0$ or $m(A)=1$. (ii) For any sequence $\left\{a_{n}\right\}$ of real numbers, $a_{n} \rightarrow 0$ implies $\frac{1}{n} \sum_{k=1}^{n}\left|a_{k}\right| \rightarrow 0$ which in turn implies $\frac{1}{n} \sum_{k=1}^{n} a_{k} \rightarrow 0$, respectively, as $n \rightarrow \infty$. Therefore, "strong-mixing" implies "weak-mixing" and "weak-mixing" implies "ergodic." 
Like measure-preserving property, mixıng property is determıned by the knowledge of generating algebra. From this we can prove that the one-sided shift transformation defined by the following is strong-mixing and so weak-mixing and ergodic.

DEFINITION 3.3. Let $Y=\{1,2, \ldots, n\}$ and $p_{\imath}$ be the measure corresponding to each $i \in Y$ such that $\sum_{t=1}^{n} p_{t}=1$. Let us define the infinite product space

$$
X=\prod_{i=1}^{\infty} X_{i}, \quad X_{i}=Y
$$

equipped with the direct product measure $m$. Then the map $S: X \rightarrow X$ defined by

$$
S\left(x_{1}, x_{2}, \ldots, x_{k}, \ldots\right)=\left(x_{2}, x_{3}, \ldots, x_{k+1}, \ldots\right)
$$

is called the one-sided $\left(p_{1}, p_{2}, \ldots, p_{n}\right)$-shift transformation.

The one-sided $\left(p_{1}, p_{2}, \ldots, p_{n}\right)$-shift transformation $S$ is measure-preserving and thus the quadruple $(X, \mathcal{A}, m, S)$ is a dynamical system.

THEOREM 3.2. The one-sided $\left(p_{1}, p_{2}, \ldots, p_{n}\right)$-shift transformation $S$ is strong-mixing.

PROOF. We call the set $\cup_{k=1}^{\infty}\left\{A \subset X: A=\prod_{i=1}^{\infty} A_{l}, A_{l}=Y\right.$ for all $\left.i \geq k\right\}$ measurable rectangles in the probability space $X$. The set of all measurable rectangles in the space $X$ creates a generating algebra. We denote it by $\mathscr{A}^{*}$. From the above remark, we need to show the strong-mixing property for this algebra. For arbitrary $A, B \in \mathcal{A}^{*}$, there exists a positive $p$ such that if $k \geq p$, then

$$
m\left(T^{-k} A \cap B\right)=m\left(T^{-k} A\right) m(B)=m(A) m(B)
$$

and thus we have

$$
\frac{1}{n} \sum_{k=0}^{n-1} m\left(T^{-k} A \cap B\right)=\frac{1}{n} \sum_{k=0}^{n-1} m\left(T^{-k} A \cap B\right)+\left(1-\frac{p}{n}\right) m(A) m(B)
$$

which converges to $m(A) m(B)$ as $n \rightarrow \infty$. The theorem, therefore, is proved.

\section{ISOMORPHISM OF MEASURE-PRESERVING TRANSFORMATIONS}

The content of this section is our main result. In previous section, we show that the one-sided $\left(p_{1}, p_{2}, \ldots, p_{n}\right)$-shift transformation is ergodic, weak-mixing and strong-mixing. In this section, we define a measure-preserving transformation on the Cantor measure space, i.e., the Cantor ternary set, and then show that this transformation is isomorphic to the one-sided $\left(\frac{1}{2}, \frac{1}{2}\right)$-shift transformation defined on the product space $X$ (see Definition 3.3). The Cantor measure space together with the one-sided shift transformation, therefore, becomes a dynamical system having the three mixing properties.

THEOREM 4.1. Suppose that $\left(C, \mathcal{F}, \mu_{F}\right)$ is a probability space, where $C$ is the Cantor ternary set, $\mathcal{F}$ is a $\sigma$-algebra generated by finite disjoint unions of sets of the form $[a, b) \cap C, 0 \leq a<b \leq 1$, and $\mu_{F}$ is the Cantor measure. Then the function $T: C \rightarrow C$ defined by

$$
T\left(2 \sum_{n=1}^{\infty} \frac{a_{n}}{3^{n}}\right)=2 \sum_{n=1}^{\infty} \frac{a_{n+1}}{3^{n}}, \quad a_{n} \in\{0,1\}
$$

is a measure-preserving transformation.

PROOF. We can use the form $[a, b] \cap C$ instead of $[a, b) \cap C$ as a generating algebra, denoted by $\mathcal{F}^{*}$, since the latter is a countable union of the sets of the former form. Also, without loss of generality, we can assume that $a, b \in C$, let

$$
a=2 \sum_{n=1}^{\infty} \frac{a_{n}}{3^{n}}, \quad b=2 \sum_{n=1}^{\infty} \frac{b_{n}}{3^{n}}, \quad a_{n}, b_{n} \in\{0,1\} .
$$


We shall show first the equality

$$
T^{-1}([a, b] \cap C)=\left(\left[\frac{a}{3}, \frac{b}{3}\right] \cap c\right) \cap\left(\left[\frac{a+2}{3}, \frac{b+2}{3}\right] \cap c\right)
$$

which is a disjoint union because $b<a+2$. Then obviously the right-hand side of (4.3) belongs to $\boldsymbol{F}^{*}$ so that $T$ becomes measurable. Now let $x \in[a, b] \cap C$ with $x=2 \sum_{n=1}^{\infty} \frac{1}{3^{n}}$. Then $a_{n} \leq x_{n} \leq b_{n}$ up to $n=n_{0}$ for some $n_{0}$ and $T^{-1}(x)=2 \sum_{n=1}^{\infty} \frac{l_{n-1}}{z^{n}}$, where $x_{0}$ is either 0 or 1 , and thus $T^{-1}(x)$ belongs to the right side of (4.3). For the reverse direction of subset, let $y \in\left[\frac{a}{3}, \frac{b}{3}\right] \cap C$ with $y=2 \sum_{n=1}^{\infty} \frac{h_{n}}{3^{n}}$. Then $y_{1}=0$ and $a_{n-1} \leq y_{n}<b_{n-1}$ up to $n_{0}$ for some $n_{0}$ and thus $a \leq T(y) \leq b$. Similarly, the set $\left[\frac{a+2}{3}, \frac{b+2}{3}\right] \cap c$ can be shown to be a subset of $T^{-1}([a, b] \cap C)$. Therefore the equality (4.3) is established. Next we will show that $\mu_{F}\left(T^{-1}([a, b] \cap C)\right)=\mu_{F}([a, b] \cap C)$.

$$
\begin{aligned}
\mu_{F}\left(T^{-1}([a, b] \cap C)\right) & =\mu_{F}\left(\left[\frac{a}{3}, \frac{b}{3}\right] \cap C\right)+\mu_{F}\left(\left[\frac{a+2}{3}, \frac{b+2}{3}\right] \cap C\right) \\
& =F\left(\frac{b}{3}\right)-F\left(\frac{a}{3}\right)+F\left(\frac{b+2}{3}\right)-F\left(\frac{a+2}{3}\right) \\
& =\sum_{n=1}^{\infty} \frac{b_{n}}{2^{n+1}}-\sum_{n=1}^{\infty} \frac{a_{n}}{2^{n+1}}+\left(\frac{1}{2}+\sum_{n=1}^{\infty} \frac{b_{n}}{2^{n+1}}\right)-\left(\frac{1}{2}+\sum_{n=1}^{\infty} \frac{a_{n}}{2^{n+1}}\right) \\
& =\sum_{n=1}^{\infty} \frac{b_{n}}{2^{n}}-\sum_{n=1}^{\infty} \frac{a_{n}}{2^{n}} \\
& =F(b)-F(a) .
\end{aligned}
$$

The theorem, therefore, is proved.

DEFINITION 4.1. Suppose that the quadruples $\left(X_{1}, \mathcal{A}_{1}, m_{1}, T_{1}\right)$ and $\left(X_{2}, \mathcal{A}_{2}, m_{2}, T_{2}\right)$ are two dynamical systems. Then we say that $T_{1}$ is isomorphic to $T_{2}$ if there exist $M_{1} \in \mathcal{A}_{1}$ and $M_{2} \in \mathcal{A}_{2}$ with $m_{1}\left(M_{1}\right)=1$ and $m_{2}\left(M_{2}\right)=1$ with property that

$$
T_{1}\left(M_{1}\right) \subset M_{1}, \quad T_{2}\left(M_{2}\right) \subset M_{2}
$$

and there exists an invertible measure-preserving transformation $\Phi: M_{1} \rightarrow M_{2}$ such that

$$
\Phi T_{1}=T_{2} \Phi
$$

on $M_{1}$.

THEOREM 4.2. Suppose the quadruples $(X, \mathcal{A}, m, S)$ and $\left(\mathcal{C}, \mathcal{J}, \mu_{F}, T\right)$ are the dynamical systems defined in Definition 3.3 and Theorem 4.1, respectively, where $X=\prod_{t=1}^{\infty} X_{t}, X_{t}=\{0,1\}$. Then $S$ is isomorphic to $T$.

PROOF. To define an appropriate map $\Phi$, we first define the following notation: Let $\left(x_{1}, x_{2}, \ldots, x_{n}, \ldots\right)$ be an arbitrary element in $X$, i.e., $x_{n}=0$ or 1 for any $n=1,2, \ldots K_{1}\left(x_{1}\right)$ denotes the closed interval of the left side of the first removed open interval $\left(\frac{1}{3}, \frac{2}{3}\right)$ if $x_{1}=0$; that of the right side of it if $x_{1}=1 . K_{2}\left(x_{1}, x_{2}\right)$ denotes the closed interval of the left (right) side of the second removed open interval contained in $K_{1}\left(x_{1}\right)$ if $x_{2}=0\left(x_{2}=1\right)$. Inductively, $K_{n}\left(x_{1}, x_{2}, \ldots, x_{n}\right)$ is the closed interval of the left (right) side of the $n$-th removed open interval contained in $K_{n-1}\left(x_{1}, x_{2}, \ldots, x_{n-1}\right)$ if $x_{n}=0\left(x_{n}=1\right)$. We observe that, for any given $\left(x_{1}, x_{2}, \ldots, x_{n}, \ldots\right) \in X, \cap_{n=1}^{\infty} K_{n}\left(x_{1}, x_{2}, \ldots, x_{n}\right)$ is a singleton subset of the Cantor ternary set $C$ and furthermore the set $C$ consists of the collection of these singleton set over the space $X$ : 


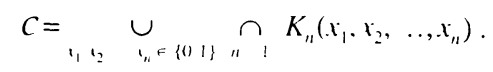

Now, in view of Definition 4.1, we take $X$ and $C$ as $M_{1}$ and $M_{2}$, respectively, and define the map $\Phi: X \rightarrow$ c by

$$
\Phi\left(x_{1}, x_{2}, \ldots, x_{n}, \ldots\right)=\overbrace{n=1}^{\infty} K_{n}\left(x_{1}, x_{2}, \ldots, x_{n}\right),
$$

where we actually take the element of the set, not set itself, for the right hand side of (4.7). We observe that $K_{n}\left(x_{1}, x_{2}, \ldots x_{n}\right)$ is the closed interval whose left end point is $2 \sum_{k=1}^{\infty} \frac{1}{l^{4}}, x_{n+1}=1_{n+2}=\ldots=0$. and whose right end point $1<2 \sum_{k=1}^{\infty} \frac{1}{3^{k}}, x_{n+1}=x_{n+2}=. .=1$ and $\Phi\left(x_{1}, x_{2}, \quad, x_{n}, \ldots\right)$ is the sance as $2 \sum_{n=1}^{\infty} \frac{i_{n}}{2^{n}}$. Then $\Phi$ is continuous, increasing and invertible. Also, it satisfics

$$
\Phi S=T \Phi
$$

on $X$. We shall show that $\Phi$ is measure-preserving. It suffices to show that, for every $a, b \in[0,1]$ with $a<b, \Phi^{-1}([a, b] \cap C) \in \mathcal{A}$ and $m\left(\Phi^{-1}([a, b] \cap C)\right)=v([a, b] \cap \mathcal{C})$. If $a \notin C$ and $b \notin C$, then at some (first) finite step, say $m$, of removing open intervals both $a$ and $b$ are removed.

$$
[a, b] \cap C=\bigcup_{i=1}^{r^{(a, b, i)}}\left(K_{m}\left(a_{i,}, a_{t,}, \ldots, a_{t_{m}}\right) \cap C\right),
$$

where $a_{t_{1}}, a_{t,}, \ldots, a_{t_{m}} \in\{0,1\}, 1 \leq i \leq p(a, b)$, and the positive integer $p(a, b)$ denotes the number of the closed intervals of the form $K_{m}\left(a_{t_{1}}, a_{t_{2}}, \ldots, a_{t_{m}}\right)$ which lic between $a$ and $b$. Therefore, from the way of the construction of $K_{n}\left(x_{1}, x_{2}, \ldots, \lambda_{1}\right)$, we obtain that $\left.\Phi^{-1}(\mid a, b] \cap C\right) \in \mathcal{A}$ and $m\left(\Phi^{-1}([a, b] \cap C)=p(a, b)\left(\frac{1}{2}\right)^{m}=\mu_{F}([a, b] \cap C)\right.$. In the case that $a \in C$ or $b \in C$; we take a sequence of elements in the complement of $C$ that converges to $a$ or $b$. The existence of the sequence comes from the property that $C$ is nowhere dense. From the first case and the continuty of the map $\Phi$, the desired result will follow.

\section{REFERENCES}

[1] WALTERS, P., Ergodic Theory-Introductory Lectures, Springer-Verlag, Berlın- HeidelbergNew York, 1975.

[2] SINAI, Y. G., Introduction to Ergodic Theory, Princeton University Press. 1976.

[3] RIZKALLA, R. R., An orthogonal system on the construction of the generalized Cantor set, Internat. J. Math. \& Math. Sci., Vol. 16, No. 4 (1993), 737-748.

[4] HALMOS, P. R., Measure Theory, Van Nostrand, New York, 1950 


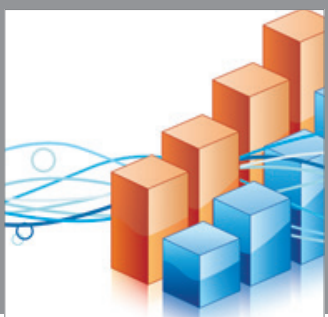

Advances in

Operations Research

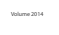

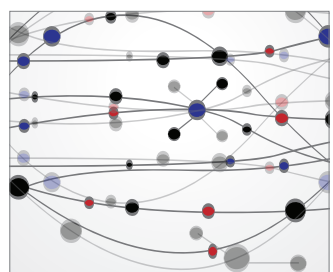

\section{The Scientific} World Journal
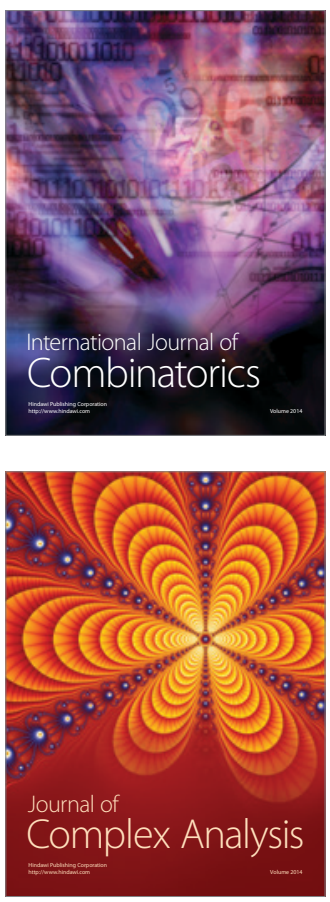

International Journal of

Mathematics and

Mathematical

Sciences
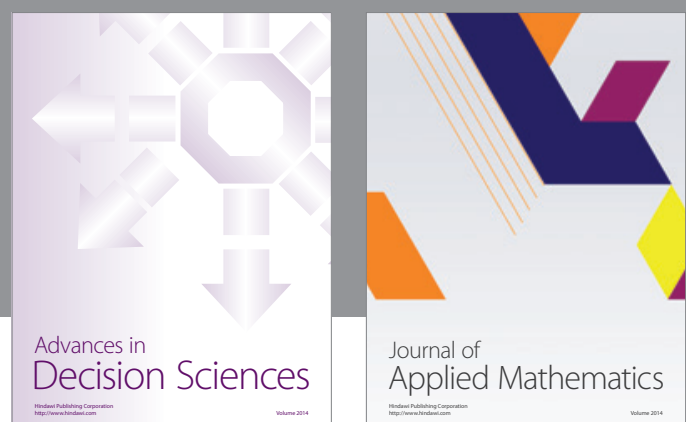

Journal of

Applied Mathematics
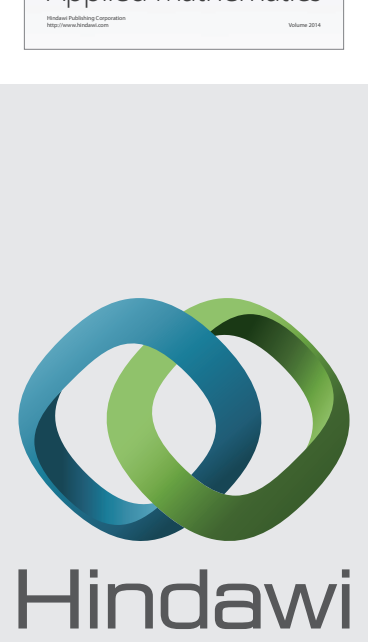

Submit your manuscripts at http://www.hindawi.com
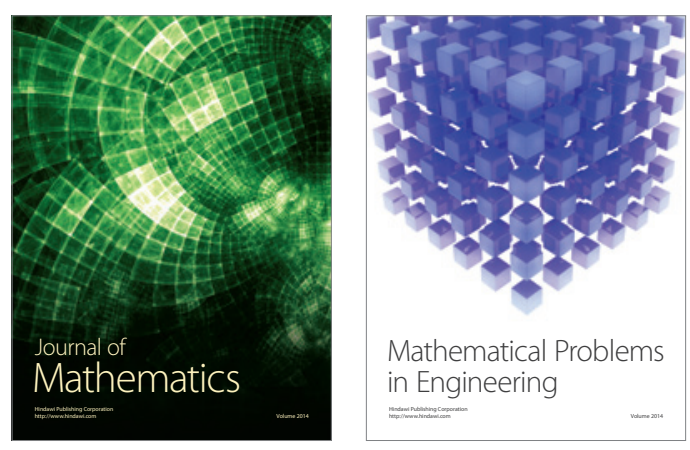

Mathematical Problems in Engineering
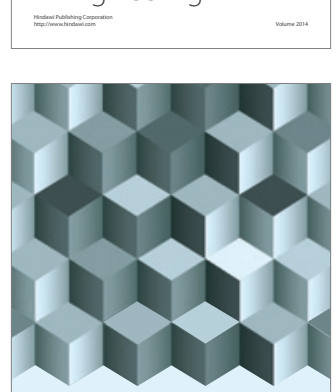

Journal of

Function Spaces
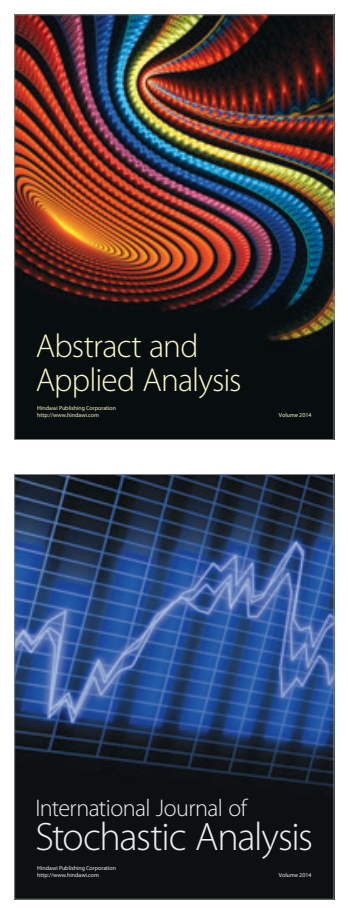

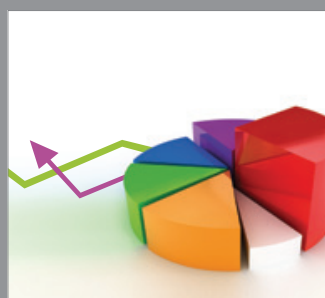

ournal of

Probability and Statistics

Promensencen
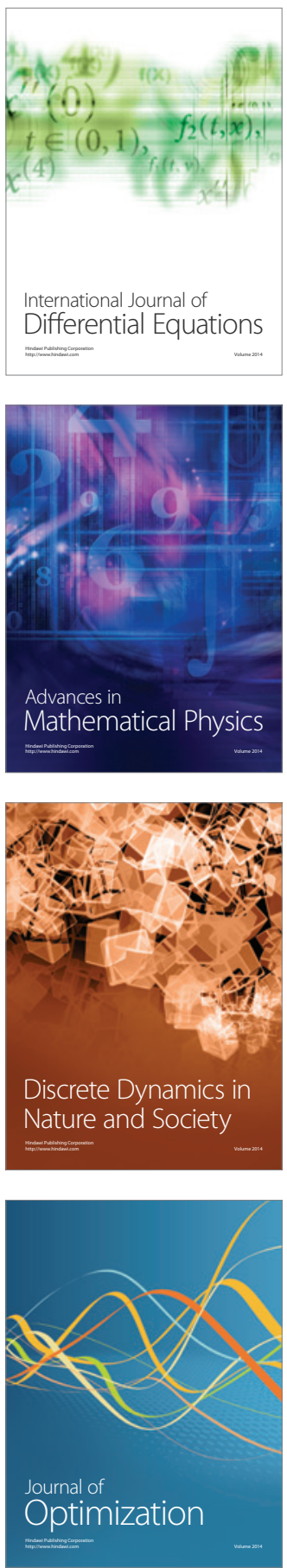